\title{
A New Lower Bound on the Number of Odd Values of the Ordinary Partition Function
}

\author{
Dennis Eichhorn \\ Department of Mathematics, University of California, Irvine, CA 92697, USA \\ deichhor@math.uci.edu
}

Received June 22, 2006

AMS Subject Classification: 05A17, 11P81, 11P82

\begin{abstract}
The parity of $p(n)$, the ordinary partition function, has been studied for at least a century, yet it still remains something of a mystery. Although much work has been done, the known lower bounds for the number of even and odd values of $p(n)$ for $n \leq N$ still appear to have a great deal of room for improvement. In this paper, we use classical methods to give a new lower bound for the number of odd values of $p(n)$.
\end{abstract}

Keywords: parity, partitions, odd values, $p(n)$

\section{Introduction and Background}

Computing the parity of specific values of $p(n)$, the ordinary partition function, has been possible since the time of Euler, but information about the overall distribution of the parity of $p(n)$ has been much more elusive. It is a long standing conjecture that $p(n)$ is odd (and even) half of the time asymptotically, but it was not until 1959 that Kolberg proved that it is both odd and even infinitely often [8]. In 1967 [11], Parkin and Shanks undertook an in-depth computational investigation of the parity of $p(n)$, and their work (including the parity of over two million values of $p(n)$ ) does support the conjecture. In 1996 [9], Ono showed that $p(n)$ is even infinitely often in every arithmetic progression, and also that it is odd infinitely often in every arithmetic progression, provided that it is at least once. In 1998 [10], Nicolas, Ruzsa, and Sárközy proved that for every $\varepsilon>0$,

$$
\#\{n \leq N: p(n) \text { is odd }\}>\sqrt{N} e^{-(\log 2+\varepsilon) \frac{\log N}{\log \log N}}
$$

for $N$ sufficiently large. They also showed that for some constant $c$,

$$
\#\{n \leq N: p(n) \text { is even }\}>c \sqrt{N}
$$

for $N$ sufficiently large. Amazingly, these were the first lower bounds that were greater than a power of $\log N$. In an appendix to [10], Serre showed that for any $t$ 
and $r$,

$$
\lim _{N \rightarrow \infty} \frac{\#\{n \leq N: p(t n+r) \text { is even }\}}{\sqrt{N}}=\infty .
$$

In 1999 [1], Ahlgren quantified Ono's work, and gave new lower bounds for the number of odd values of $p(n)$ in arithmetic progressions. The explicit bound he gave for the number of odd values of $p(n)$ is

$$
\#\{n \leq N: p(n) \text { is odd }\}>\frac{4 \sqrt{N}}{\log 8 N}
$$

for $N$ sufficiently large. The technology used in this result involves both theorems from the theory of modular forms and properties of $\ell$-adic Galois representations. In this paper, we give a better lower bound for the number of odd values of $p(n)$ using only Euler's Pentagonal Number Theorem, Jacobi's Identity, the classical formula for $r_{2}(n)$ (the number of representations of $n$ as a sum of two squares), and the prime number theorem for a single arithmetic progression.

\section{The Lower Bound}

Euler showed that the generating function for $p(n)$ is

$$
\sum_{n=0}^{\infty} p(n) q^{n}=\prod_{j=1}^{\infty} \frac{1}{1-q^{j}}
$$

In [5], it was shown that the generating function for $a_{4}(n)$ is

$$
\sum_{n=0}^{\infty} a_{4}(n) q^{n}=\prod_{j=1}^{\infty} \frac{\left(1-q^{4 j}\right)^{4}}{1-q^{j}}
$$

where $a_{4}(n)$ is the number of 4-core partitions of $n$ (a 4-core partition is a partition whose Ferrers diagram has no hook numbers divisible by 4 ; however, the combinatorics of 4-core partitions will play no role in what follows). In [6], Hirschhorn and Sellers use generating function manipulations relying only on Jacobi's Identity to show that $a_{4}(n)$ is congruent modulo 2 to $\frac{1}{8} r_{2}(8 n+5)$, where $r_{2}(n)$ is the number of representations of $n$ as a sum of two squares. Using a classical result expressing $r_{2}(n)$ as a difference of divisor functions, they determine necessary and sufficient conditions for $a_{4}(n)$ to be even, which we restate in the following way.

Lemma 2.1. (Hirschhorn and Sellers) The value of $a_{4}(n)$ is odd if and only if $8 n+$ $5=p^{e} M^{2}$ for some integers $p$, $e$, and $M$, with $p$ prime, $e \equiv 1(\bmod 4)$, and $p \nmid M$.

We can now use the prime number theorem for the arithmetic progression 5 $(\bmod 8)$ to count the odd values of $a_{4}(n)$.

Lemma 2.2. The number of odd values of $a_{4}(n)$ with $n \leq N$ is $\left(\frac{\pi^{2}}{4}+o(1)\right) \frac{N}{\log N}$. 
Proof. Let $T_{N, M}$ be $\left\{n \leq N: 8 n+5=p M^{2}\right.$ for some prime $\left.p \nmid M\right\}$ (notice here that $M$ must be odd and $p \equiv 5(\bmod 8))$. For each fixed odd $M$, fewer than $\log M$ primes $p \mid M$, so by the prime number theorem for the arithmetic progression $5(\bmod 8)$,

$$
\# T_{N, M}=\left(\frac{1}{4}+o(1)\right) \frac{8 N+5}{M^{2}\left[\log (8 N+5)-\log \left(M^{2}\right)\right]}=\frac{(2+o(1)) N}{M^{2} \log N},
$$

where here we have adopted the usual convention that " $o(1)$ " denotes some function that tends to zero as $N \rightarrow \infty$. Taking the union over odd $M$ up to $N^{1 / 3}$ and recalling that the sum of the reciprocals of the odd squares is $\frac{\pi^{2}}{8}$, we have

$$
\# \bigcup_{\substack{M \text { odd } \\ M \leq N^{1 / 3}}} T_{N, M}=\left(\frac{\pi^{2}}{4}+o(1)\right) \frac{N}{\log N} .
$$

Observe that each nonempty $T_{N, M}$ with $M>N^{1 / 3}$ has size less than $8 N^{1 / 3}$; there are fewer than $\sqrt{N}$ such sets, so their union has size less than $8 N^{5 / 6}$. Also observe that there are fewer than $N^{1 / e+1 / 2}$ positive integers of the form $p^{e} M^{2}$ less than $N$. Combining these observations with (2.1) and Lemma 2.1, we have

$$
\#\left\{n \leq N: a_{4}(n) \text { is odd }\right\}=\left(\frac{\pi^{2}}{4}+o(1)\right) \frac{N}{\log N}
$$

as desired.

With Lemma 2.2 in place, we are now able to prove our main theorem.

Theorem 2.3. The number of odd values of $p(n)$ is bounded below as follows:

$$
\#\{n \leq N: p(n) \text { is odd }\} \geq\left(\frac{\pi^{2} \sqrt{6}}{4}+o(1)\right) \frac{\sqrt{N}}{\log N} .
$$

Proof. Since we are only concerned with parity, all of our power series calculations will be in $\mathbb{F}_{2}[[q]]$. This being the case, notice that $(1-z)^{4}=\left(1-z^{2}\right)^{2}=\left(1-z^{4}\right)$ by the "mod 2 binomial theorem" for any $z \in \mathbb{F}_{2}[[q]]$.

Now consider

$$
\begin{aligned}
\sum_{n=0}^{\infty} a_{4}(n) q^{n} & =\prod_{j=1}^{\infty} \frac{\left(1-q^{4 j}\right)^{4}}{1-q^{j}} \\
& =\prod_{j=1}^{\infty} \frac{\left(1-q^{16 j}\right)}{1-q^{j}} \\
& =\left(\sum_{n=0}^{\infty} p(n) q^{n}\right)\left(\sum_{m=-\infty}^{\infty} q^{8 m(3 m-1)}\right),
\end{aligned}
$$

where in the last step we have applied Euler's Pentagonal Number Theorem with $q$ replaced by $q^{16}$ to the numerator of the previous line. Notice that $\sum_{m=-\infty}^{\infty} q^{8 m(3 m-1)}$ 
has $\sqrt{N / 6}(1+o(1))$ nonzero terms up through the $q^{N}$ term. Also recall that, by Lemma $2.2, \sum_{n=0}^{\infty} a_{4}(n) q^{n}$ has $\left(\frac{\pi^{2}}{4}+o(1)\right) \frac{N}{\log N}$ nonzero terms up through the $q^{N}$ term. Since every nonzero term on the left-hand side of (2.2) must come from an odd value of $p(n)$ coupled with a nonzero term of $\sum_{m=-\infty}^{\infty} q^{8 m(3 m-1)}$, we have

$$
\#\{n \leq N: p(n) \text { is odd }\} \cdot \sqrt{\frac{N}{6}}(1+o(1)) \geq\left(\frac{\pi^{2}}{4}+o(1)\right) \frac{N}{\log N},
$$

and our result follows.

In [1], Ahlgren shows that for $r=0,1,2$, or $3, \#\{n \leq N: n \equiv r(\bmod 4), p(n)$ is odd $\}$ is greater than $\frac{\sqrt{N}}{\log 8 N}$. Combining these four arithmetic progressions, his lower bound on the number of odd values of $p(n)$ with $n \leq N$ is $\frac{4 \sqrt{N}}{\log 8 N}$, so we see that Theorem 2.3 improves this bound by a bit more than a factor of $3 / 2$. At first glance, one might think that we have sacrificed the information in arithmetic progressions with modulus 4 to achieve a better lower bound; however, a careful examination of the proof of Theorem 2.3 reveals that in fact, the opposite is true. Since the second sum on the right-hand side of (2.2) is a power series in $q^{16}$, we actually have information refined into every arithmetic progression with modulus 16.

Theorem 2.4. For any integer $0 \leq r<16$,

$$
\#\{n \leq N: n \equiv r(\bmod 16), p(n) \text { is odd }\} \geq\left(\frac{\pi^{2} \sqrt{6}}{64}+o(1)\right) \frac{\sqrt{N}}{\log N} .
$$

Proof. By reasoning as in Lemma 2.2, $\sum_{n=0}^{\infty} a_{4}(16 n+r) q^{16 n+r}$ has at least $\left(\frac{\pi^{2}}{64}+\right.$ $o(1)) \cdot \frac{N}{\log N}$ nonzero terms up through the $q^{N}$ term (notice here that primes $p \equiv 8 r+5$ $(\bmod 128)$ take the place of primes $p \equiv 5(\bmod 8)$ in Lemma 2.2$)$. Now, since the second sum on the right-hand side of (2.2) is actually a power series in $q^{16}$, contributions to $\sum_{n=0}^{\infty} a_{4}(16 n+r) q^{16 n+r}$ can only come from odd values of $p(16 n+r)$ coupled with nonzero terms of $\sum_{m=-\infty}^{\infty} q^{8 m(3 m-1)}$. Thus,

$$
\#\{n \leq N: n \equiv r \quad(\bmod 16), p(n) \text { is odd }\} \cdot \sqrt{\frac{N}{6}}(1+o(1)) \geq\left(\frac{\pi^{2}}{64}+o(1)\right) \frac{N}{\log N},
$$

and our result follows.

It is worth mentioning here that for each $r,\{p(16 n+r)\}$ was first shown to contain both infinitely many odd values and infinitely many even values by Hirschhorn and Subbarao in [7]. Our (2.2) coupled with the first step of the analysis in [6] appeared first in [7] as the key ingredient of their proof.

\section{Other Results}

One nice feature of this new lower bound for the number of odd values of $p(n)$ is that the proof contains a certain amount of concrete information that addresses not only 
the number of odd values, but also their location. Although we have not explicitly exhibited an infinite set of $n$ for which $p(n)$ is odd, we still can draw some conclusions about where a certain number of odd values must be. Also, since Lemma 2.1 tells us explicitly when $a_{4}(n)$ is odd, we can use that information to draw conclusions about partition functions other than $p(n)$, which would not be possible if we had only the result of Theorem 2.3 in isolation. In this section, we demonstrate these nice features by providing a few further results that follow from arguments given in the proof of Theorem 2.3.

In Theorem 3.1, we draw a slightly stronger conclusion than the final statement of Theorem 2.4. As it turns out, for every positive integer $n$, we know the parity of the number of odd values in $\{p(n-8 m(3 m-1)): m \in \mathbb{Z}\}$. Let $A_{4}$ be the set of all integers $n$ such that $a_{4}(n)$ is odd (this set is given explicitly in Lemma 2.1).

Theorem 3.1. If $n \in A_{4}$, then

$$
\{p(n-8 m(3 m-1)): m \in \mathbb{Z}\} \text { contains an odd number of odd values }
$$

otherwise, it contains an even number of odd values.

Proof. This follows immediately from equating coefficients of $q^{n}$ on the left- and right-hand sides of (2.2), and recalling that this is an identity in $\mathbb{F}_{2}[[q]]$.

Now, Theorem 2.3 could be viewed as a corollary of Theorem 3.1 that reflects the fact that if $\{p(n-8 m(3 m-1)): m \in \mathbb{Z}\}$ contains an odd number of odd values, it must contain at least one.

Our next result gives a lower bound on the number of odd values of $p_{a}(n)$, where $p_{a}(n)$ is the number of partitions of $n$ with no parts of size $a$. This is made possible by a somewhat surprising appeal to upper bounds for the number of prime 2-tuples.

Theorem 3.2. For any positive integer $a$,

$$
\#\left\{n \leq N: p_{a}(n) \text { is odd }\right\} \geq\left(\frac{\left(16-\pi^{2}\right) \sqrt{6}}{2}+o(1)\right) \frac{\sqrt{N}}{\log N} .
$$

Proof. Notice that the generating function for $p_{a}(n)$ is the generating function for $p(n)$ multiplied by $\left(1-q^{a}\right)$. Thus, multiplying both sides of (2.2) by $\left(1-q^{a}\right)$, we have

$$
\left(1-q^{a}\right) \sum_{n=0}^{\infty} a_{4}(n) q^{n}=\left(\sum_{n=0}^{\infty} p_{a}(n) q^{n}\right)\left(\sum_{m=-\infty}^{\infty} q^{8 m(3 m-1)}\right) .
$$

Now we see that a lower bound like the one we gave for $p(n)$ will also hold for $p_{a}(n)$, provided that multiplication by $\left(1-q^{a}\right)$ does not thin the power series on the left-hand side of (3.1) too severely.

The powers of $q$ that occur on the left-hand side of (3.1) will be exactly the symmetric difference of $A_{4}$ and the translation of $A_{4}$ upwards by $a$ (we will denote this symmetric difference as " $A_{4} \triangle\left(A_{4}+a\right)$ "). If we consider the subset $P_{4}$ of $A_{4}$ that consists of all elements of the form $\frac{p-5}{8}$ where $p$ is prime, then $P_{4}$ has $(2+o(1)) \frac{N}{\log N}$ 
elements up to $N$. Of course, $P_{4}+a$ also has $(2+o(1)) \frac{N}{\log N}$ elements up to $N$, so that $P_{4} \triangle\left(P_{4}+a\right)$ has $(4+o(1)) \frac{N}{\log N}-R(N)$ elements up to $N$, where $R(N)$ is twice the number of elements in $P_{4} \cap\left(P_{4}+a\right)$ up to $N$. If $n \in P_{4} \cap\left(P_{4}+a\right)$, then both $8 n+5$ and $8 n+5-8 a$ are prime. However, by bounds on the number of prime 2-tuples of the form $(8 n+5,8 n+5-8 a)$ (see [4], for example), we know that the relative density of these 2-tuples amongst the primes is zero, and so the size of $P_{4} \cap\left(P_{4}+a\right)$ is $o\left(\frac{N}{\log N}\right)$. Thus, $P_{4} \triangle\left(P_{4}+a\right)$ has $(4+o(1)) \frac{N}{\log N}$ elements up to $N$. Since $A_{4} \backslash P_{4}$ and $\left(A_{4}+a\right) \backslash\left(P_{4}+a\right)$ each have $\left(\frac{\pi^{2}}{4}-2+o(1)\right) \frac{N}{\log N}$ elements up to $N$, and

$$
A_{4} \triangle\left(A_{4}+a\right) \supseteq P_{4} \triangle\left(P_{4}+a\right) \backslash\left[\left(\left[A_{4} \backslash P_{4}\right] \cap\left[A_{4}+a\right]\right) \cup\left(\left[\left(A_{4}+a\right) \backslash\left(P_{4}+a\right)\right] \cap A_{4}\right)\right],
$$

we have that the number of elements in $A_{4} \triangle\left(A_{4}+a\right)$ up to $N$ is at least $\left(8-\frac{\pi^{2}}{2}+\right.$ $o(1)) \frac{N}{\log N}$. Following the analysis in the proof of Theorem 2.3, we have

$$
\#\left\{n \leq N: p_{a}(n) \text { is odd }\right\} \geq\left(\frac{\left(16-\pi^{2}\right) \sqrt{6}}{2}+o(1)\right) \frac{\sqrt{N}}{\log N}
$$

as desired.

We could give a much sharper bound on the amount of cancelation that occurs in the product on the left-hand side of (3.1), and give a better lower bound for $\#\{n \leq$ $N: p_{a}(n)$ is odd $\}$. However, we have given a smaller lower bound here in order to more quickly highlight one way in which information about the distribution of primes can be applied to this question. Of course, this result may also be refined into arithmetic progressions with modulus 16 as was done in Theorem 2.4. In addition, we can get similar results for $p_{S}(n)$ for any finite set $S$, where $p_{S}(n)$ is the number of partitions of $n$ with no parts from $S$.

\section{Conclusion and Further Study}

If we analyze the proof of Theorem 2.3, we see that the key ingredient for our result was the ability to express a power series in $\mathbb{F}_{2}[[q]]$ with a known thickness as a product of the generating function for $p(n)$ and a very sparse series. It is certainly possible that we may be able to further improve our lower bound by considering other such products. In Theorem 3.2, we were able to extend our results to another family of partition functions. It is also possible that other families of partition functions can be treated using this method. We save these explorations for study in a future project.

Acknowledgments. The author would like to thank Bruce Berndt, Ae Ja Yee, and Alexandru Zaharescu for their outstanding work in [2] and [3]. Their two papers are essential reading for anyone genuinely interested in the parity of partition functions, and it was their contributions that inspired the author to revisit the parity of $p(n)$. Also, the author would like to thank the referee for a very thorough and quick treatment of the first version of this paper. 


\section{References}

1. Ahlgren, S.: Distribution of parity of the partition function in arithmetic progressions. Indag. Math. (N.S.) 10, 173-181 (1999)

2. Berndt, B.C., Yee, A.J., Zaharescu, A.: On the parity of partition functions. Internat. J. Math. 14, 437-459 (2003)

3. Berndt, B.C., Yee, A.J., Zaharescu, A.: New theorems on the parity of partition functions. J. Reine Angew. Math. 566, 91-109 (2004)

4. Bombieri, E., Davenport, H.: Small differences between prime numbers. Proc. Roy. Soc. Ser. A 293, 1-18 (1966)

5. Garvan, F., Kim, D., Stanton, D.: Cranks and $t$-cores. Invent. Math. 101, 1-17 (1990)

6. Hirschhorn, M.D., Sellers, J.A.: Two congruences involving 4-cores. Electron. J. Combin. 3, \#10 (1996)

7. Hirschhorn, M.D., Subbarao, M.V.: On the parity of $p(n)$. Acta Arith. 50, 355-356 (1988)

8. Kolberg, O.: Note on the parity of the partition function. Math. Scand. 7, 377-378 (1959)

9. Ono, K.: Parity of the partition function in arithmetic progressions. J. Reine Angew. Math. 472, 1-15 (1996)

10. Nicolas, J.-L., Ruzsa, I.Z., Sárközy, A.: On the parity of additive representation functions. J. Number Theory 73, 292-317 (1998)

11. Parkin, T.R., Shanks, D.: On the distribution of parity in the partition function. Math. Comp. 21, 466-480 (1967)

Open Access This article is distributed under the terms of the Creative Commons Attribution Noncommercial License which permits any noncommercial use, distribution, and reproduction in any medium, provided the original author(s) and source are credited. 